\title{
Pre-wavelet bases in Lebesgue spaces
}

\section{Chiou-Yueh Gun ${ }^{1}$, Kai-Cheng Wang ${ }^{2^{*}}$ (D), Chi-I Yang ${ }^{2}$ and Kuei-Fang Chang ${ }^{3}$}

"Correspondence:
gtotony98@gmail.com
2Ph.D. Program in Mechanical and
Aeronautical Engineering,
Feng-Chia University, Wenhwa
Road, Taichung, 40724, Taiwan
Full list of author information is
available at the end of the article

available at the end of the article

\begin{abstract}
Under the decay condition, we have constructed the dual wavelet basis of a pre-wavelet basis. The frame operators of both bases are bijective on Lebesgue spaces. Both bases are also unconditional bases for Lebesgue spaces.

MSC: Primary 42C40; secondary 42C15

Keywords: bijectivity; Calderón-Zygmund decomposition theorem; frame; Riesz basis; semi-orthogoal; unconditional basis; wavelet; Wiener's lemma
\end{abstract}

\section{Introduction}

This study was motivated by the following two facts. There exists a compactly supported, smooth Riesz wavelet basis such that the corresponding frame operator is bijective on $L^{2}(\mathbb{R})$, but not on $L^{p}(\mathbb{R})$ for any specified $1<p<2[1]$. However, a frame operator is a composition of a pre-frame operator and the adjoint operator of the pre-frame operator. One therefore wonders whether the Riesz wavelet basis is complete in $L^{p}(\mathbb{R}), 1<p<2$. The second one was found in [2], p.130,136, in which a Riesz wavelet basis with good behavior was found to maybe be not complete in $L^{p}(\mathbb{R}), 2<p<\infty$. A more detailed understanding of such wavelets can be found in Remark 2.2.

This study confirms the bijectivity of the pre-wavelet (semi-orthogonal wavelet) frame operator on $L^{p}(\mathbb{R}), 1<p<\infty$ (Theorem 3.4). Due to bijectivity of the frame operator, it is certain that the pre-frame operator of the wavelet basis is surjective on $L^{p}(\mathbb{R}), 1<p<\infty$. The keys for proving Theorem 3.4 are that the dual wavelet basis of the pre-wavelet basis has been constructed, and it has the same decay as the basis. In contrast, the two Riesz wavelet bases discussed earlier had only put emphasis on the wavelet behavior, but they ignored the behavior of a dual basis. It may yield negative results in the completeness of the wavelet bases in Lebesgue spaces.

This paper is organized as follows. In Section 2, we give the relevant theory of wavelet frames. In Section 3, we prove Lemma 3.1 and Theorems 3.2-3.4. Finally, we give further comments and examples in Section 4.

\section{Preliminaries and notations}

The bases in this paper are Schauder bases. Let $\mathcal{B}$ be a Banach space. A sequence $\left\{f_{k}\right\} \subset \mathcal{B}$ is called a basis if for all $f \in \mathcal{B}$, there exists unique sequence of scalars $\left\{a_{k}\right\}$ such that

$$
f=\sum_{k} a_{k} f_{k}
$$

(c) 2015 Gun et al. This article is distributed under the terms of the Creative Commons Attribution 4.0 International License (http://creativecommons.org/licenses/by/4.0/), which permits unrestricted use, distribution, and reproduction in any medium, provided you give appropriate credit to the original author(s) and the source, provide a link to the Creative Commons license, and indicate if changes were made. 
A basis $\left\{f_{k}\right\}$ is unconditional in a Banach space if any convergent series $\sum_{k} a_{k} f_{k}$ converges unconditionally; that is, the series $\sum_{k} a_{\pi(k)} f_{\pi(k)}$ converges to the same limit for all permutations $\pi$ of $\mathbb{N}$ (or $\mathbb{Z}$ ). It is well known that unconditional bases can be characterized as follows.

Theorem 2.1 ([3], Theorem 16.1) Let $\left\{f_{n}\right\}$ be a basis in a Banach space. Then the following properties are equivalent.

(1) $\left\{f_{n}\right\}$ is unconditional.

(2) There exists a positive constant $C$ such that, for all $n$, for all $\epsilon_{i}= \pm 1$, and for all scalars $a_{1}, a_{2}, \ldots, a_{n}$,

$$
\left\|\sum_{i=1}^{n} \epsilon_{i} a_{i} f_{i}\right\| \leq C\left\|\sum_{i=1}^{n} a_{i} f_{i}\right\| .
$$

The Wiener amalgam space $W\left(L^{\infty}, l^{1}\right)$ was introduced by Wiener in connection with the Tauberian theorem [4]. The definition of $W\left(L^{\infty}, l^{1}\right)$ is given as follows. A measurable function $f$ belongs to $W\left(L^{\infty}, l^{1}\right)$ if it satisfies

$$
\|f\|_{W\left(L, l^{1}\right)}:=\sum_{k \in \mathbb{Z}} \sup _{[0,1)}|f|(x+k)<\infty
$$

Equation (2.1) leads to $f \in L^{p}(\mathbb{R})$, for all $1 \leq p \leq \infty$. Indeed,

$$
\begin{aligned}
\int_{\mathbb{R}}|f(x)|^{p} d x & \leq \int_{0}^{1}\left(\sum_{k \in \mathbb{Z}}|f|(x+k)\right)^{p} d x \\
& \leq\left(\sum_{k \in \mathbb{Z}} \sup _{[0,1)}|f|(x+k)\right)^{p}=\|f\|_{W\left(L^{\infty}, l^{1}\right)}^{p} .
\end{aligned}
$$

Let $Q$ be a mapping from $L^{p}(\mathbb{R})$ to $L^{q}(\mathbb{R})$, for all $1 \leq p, q \leq \infty$. Then $Q$ is of type $(p, q)[5]$ if

$$
\|Q(f)\|_{q} \leq A\|f\|_{p}, \quad f \in L^{p}(\mathbb{R})
$$

where $A$ does not depend on $f$. Similarly, $Q$ is of weak type $(p, q)$ if

$$
m\{x:|Q f(x)|>\alpha\} \leq\left(\frac{A\|f\|_{p}}{\alpha}\right)^{q}, \quad q<\infty, \alpha>0,
$$

where $A$ does not depend on $f$ or $\alpha$, and $m$ is the Lebesgue measure. We note that an operator of type $(p, q)$ is also of weak type $(p, q)$.

A family of elements $\left\{\psi_{i}: i \in \mathbb{Z}\right\}$ is called a frame for a Hilbert space $\mathcal{H}$ if there exist two constants $0<A \leq B<\infty$ such that

$$
A\|f\|^{2} \leq \sum_{i \in \mathbb{Z}}\left|\left\langle f, \psi_{i}\right\rangle\right|^{2} \leq B\|f\|^{2}, \quad \text { for all } f \in \mathcal{H} .
$$

The numbers $A, B$ are called frame bounds and if $A=B$, we call this a tight frame. Frames are essentially a set of functions that span the whole space but are not necessarily linearly 
independent. Define the frame operator $S$ of $\left\{\psi_{i}: i \in \mathbb{Z}\right\}$,

$$
S f:=\sum_{i \in \mathbb{Z}}\left\langle f, \psi_{i}\right\rangle \psi_{i}
$$

and each $f \in \mathcal{H}$ has the decomposition

$$
f=\sum_{i \in \mathbb{Z}}\left\langle f, S^{-1} \psi_{i}\right\rangle \psi_{i}=\sum_{i \in \mathbb{Z}}\left\langle f, \psi_{i}\right\rangle S^{-1} \psi_{i}
$$

The series converges unconditionally in $\mathcal{H}[6]$, pp.90-91. $\left\{S^{-1} \psi_{i}: i \in \mathbb{Z}\right\}$ is called the canonical dual of $\left\{\psi_{i}: i \in \mathbb{Z}\right\}$, and every frame has its own canonical dual frame for $L^{2}(\mathbb{R})$. $T$, which is bounded; it is called the pre-frame operator or the synthesis operator. We have

$$
T: l^{2}(\mathbb{N}) \rightarrow \mathcal{H}, \quad T\left\{c_{i}\right\}_{i \in \mathbb{Z}}:=\sum_{i \in \mathbb{Z}} c_{i} \psi_{i}
$$

$S=T T^{*}$. Both $S$ and $S^{-1}$ are of type (2,2), bounded, invertible, self-adjoint, and positive [6], p.90. $\left\{S^{-1} \psi_{i}: i \in \mathbb{Z}\right\}$ is also a frame in $\mathcal{H}$ and its frame operator is $S^{-1}$. The canonical dual frame $\left\{S^{-1} \psi_{i}: i \in \mathbb{Z}\right\}$ of a tight frame $\left\{\psi_{i}: i \in \mathbb{Z}\right\}$ is simply $\left\{\frac{1}{A} \psi_{i}: i \in \mathbb{Z}\right\}$.

Riesz bases are exact frames which obey the linear independence property, and their bounds coincide with the frame bounds. $\left\{\psi_{i}: i \in \mathbb{Z}\right\}$ is a Riesz basis for $\mathcal{H}$ if there exist constants $A, B>0$ such that for every finite scalar $\left\{c_{i}\right\}$ one has

$$
A \sum_{i}\left|c_{i}\right|^{2} \leq\left\|\sum_{i} c_{i} \psi_{i}\right\|_{2}^{2} \leq B \sum_{i}\left|c_{i}\right|^{2} .
$$

The dual basis of a Riesz basis is, again, a Riesz basis and is equal to $\left\{S^{-1} \psi_{i}\right\}$ and

$$
\left\langle\psi_{i}, S^{-1} \psi_{j}\right\rangle=\delta_{i j}
$$

A frame which is not a Riesz basis is said to be overcomplete, and thus, for overcomplete frames, the coefficients in the series expansion of $f \in \mathcal{H}$ are not unique.

We next turn to wavelets. Throughout this paper, we used the following notation:

$$
\psi_{j, k}(x):=D^{j} T_{k} \psi(x)=2^{j / 2} \psi\left(2^{j} x+k\right)
$$

where $D^{j}(\cdot)(x):=2^{j / 2}(\cdot)\left(2^{j} x\right), T_{k}(\cdot)(x):=(\cdot)(x+k)$. The affine wavelet frame system for $L^{2}(\mathbb{R})$ generated by $\psi$ is defined as $\left\{\psi_{j, k}: j, k \in \mathbb{Z}\right\}$, and it is a frame for $L^{2}(\mathbb{R})$. One can show that

$$
S^{-1} D^{j} T_{k} \psi=D^{j} S^{-1} T_{k} \psi
$$

However, in general [6], p.276,

$$
D^{j} S^{-1} T_{k} \psi \neq D^{j} T_{k} S^{-1} \psi
$$

In this case, we say that the canonical dual frame $\left\{S^{-1} \psi_{j, k}\right\}$ of $\left\{\psi_{j, k}\right\}$ does not have the wavelet structure. $\left\{\psi_{j, k}\right\}$ and $\left\{S^{-1} \psi_{j, k}\right\}$ are a pair of biorthogonal (Riesz) wavelet bases [7] 
for $L^{2}(\mathbb{R})$ if $\left\{S^{-1} \psi_{j, k}\right\}$ has the wavelet structure. Listed below (Remark 2.2) are a number of details that require attention.

\section{Remark 2.2}

(1) To avoid confusion, we denote by $H$ the frame operator of $\left\{S^{-1} \psi_{j, k}\right\}$. Notice that $H$ is bounded, invertible $(S H=H S=I)$, self-adjoint, and positive on $L^{2}(\mathbb{R})$ [6], p.90.

(2) If the frame operator of a frame is bijective on $L^{p}(\mathbb{R})$, then bijectivity will guarantee the pre-frame operator to be surjective. Therefore, bijectivity guarantees that each function $f$ in $L^{p}(\mathbb{R})$ has an expansion in terms of the wavelet.

(3) There is an important function which comes from [8], [6], p.274, [9, 10]. Let $\left\{\psi_{j, k}\right.$ : $j, k \in \mathbb{Z}\}$ be an orthonormal basis for $L^{2}(\mathbb{R})$. Given $0<\epsilon<1$, we define a function $\theta$ by

$$
\theta:=\psi+\epsilon D^{1} \psi
$$

$\left\{\theta_{j, k}: j, k \in \mathbb{Z}\right\}$ is a Riesz basis, but its canonical dual basis does not have a wavelet structure. Therefore, a function $\tilde{\theta}$ such that $\left\{\theta_{j, k}\right\}$ and $\left\{\widetilde{\theta}_{j, k}\right\}$ are a pair of biorthogonal Riesz wavelet bases does not exist. However, $S^{-1} \theta$ is not always compactly supported even when $\theta$ has compact support [6], p.276. Even when $\theta$ belongs to the Schwartz class, $S^{-1} \theta_{j, 0}$ does not belong to $L^{p}$ for small $p-1>0$ [9]. Moreover, Tao pointed out that the frame operator of $\left\{\theta_{j, k}\right\}$ with 'good' conditions does not necessarily come with the bijectivity [1]. This leads to the observation that continuously strengthening restrictions on the wavelets may not necessarily provide effectively guaranteed behavior of dual bases.

Finally, we say that $\psi$ satisfies condition $\mathcal{M}$ if

$$
\int_{0}^{\infty}\left[\log _{2}(1+x)\right] \varphi(x) d x<\infty
$$

where $\varphi(x):=\sup _{x \leq|y|}|\psi|(y), x \geq 0$ is named minimum radical dominated function of $\psi$, and $\mathcal{F}_{\psi}:=\left\{\psi_{j, k}: \psi \in \mathcal{M}, j, k \in \mathbb{Z}\right\}$ is a Riesz basis for $L^{2}(\mathbb{R})$ with $\psi \in \mathcal{M}$.

\section{Proofs and results}

The proofs of Lemma 3.1 and Theorem 3.2 are based on the Calderón-Zygmund decomposition theorem [5]. For all $f \in L^{1} \cap L^{2}(\mathbb{R})$ and $\alpha>0$, there exists a collection $\Omega \subset \mathbb{Z}^{2}$ such that the intervals $\left\{I_{m, n}\right\}_{(m, n) \in \Omega}$ are disjoint, $I_{m, n}:=\left[2^{-m} n, 2^{-m}(n+1)\right)$,

$$
|f(x)| \leq \alpha
$$

almost everywhere on $F:=\mathbb{R} \backslash \bigcup_{(m, n) \in \Omega} I_{m, n}$, and also, for all $(m, n) \in \Omega$,

$$
\alpha<2^{m} \int_{I_{m, n}}|f| \leq 2 \alpha .
$$

Therefore,

$$
\sum_{(m, n) \in \Omega} \alpha 2^{-m}<\|f\|_{1}
$$


Let $\mathcal{F}_{\psi}$ be a Riesz basis for $L^{2}(\mathbb{R})$. We denote by $P_{m}$ the projection onto a subspace of $\mathcal{V}_{m}:=\overline{\operatorname{span}}\left\{S^{-1} \psi_{j, k}: j, k \in \mathbb{Z}, j<m\right\}$,

$$
P_{m}(f):=\sum_{\substack{j, k \in \mathbb{Z} \\ j<m}}\left\langle f, \psi_{j, k}\right\rangle S^{-1} \psi_{j, k},
$$

for all $f \in L^{1} \cap L^{2}(\mathbb{R})$. For all $f \in L^{1} \cap L^{2}(\mathbb{R})$, we set

$$
g:=f \chi_{F}+\sum_{(m, n) \in \Omega} P_{m}\left(f \chi_{I_{m, n}}\right)
$$

producing

$$
\begin{aligned}
h & :=f-g=\sum_{(m, n) \in \Omega}\left[f \chi_{I_{m, n}}-P_{m}\left(f \chi_{I_{m, n}}\right)\right] \\
& =\sum_{(m, n) \in \Omega}\left[\sum_{j, k \in \mathbb{Z}}\left\langle f \chi_{I_{m, n}}, \psi_{j, k}\right\rangle S^{-1} \psi_{j, k}-P_{m}\left(f \chi_{I_{m, n}}\right)\right] \\
& =\sum_{(m, n) \in \Omega} \sum_{\substack{j, k \in \mathbb{Z} \\
j \geq m}}\left\langle f \chi_{I_{m, n}}, \psi_{j, k}\right\rangle S^{-1} \psi_{j, k}, \\
S h & =\sum_{j, k \in \mathbb{Z}}\left\langle\sum_{(m, n) \in \Omega} \sum_{j^{\prime}, k^{\prime} \in \mathbb{Z}}\left\langle f \chi_{I_{m, n}}, \psi_{j^{\prime}, k^{\prime}}\right\rangle S^{-1} \psi_{j^{\prime}, k^{\prime}}, \psi_{j, k}\right) \psi_{j, k} \\
& =\sum_{(m, n) \in \Omega} \sum_{\substack{j, k \in \mathbb{Z} \\
j \geq m}}\left\langle f \chi_{I_{m, n}}, \psi_{j, k}\right\rangle \psi_{j, k} .
\end{aligned}
$$

Here, we would like to stress the importance of Lemma 3.1, being the foundation of the main results in this study. The proofs of Lemma 3.1 and Theorem 3.2 are based on the ideas expressed in $[11,12]$, without the unnecessary assumptions and complicated proving procedures. Most importantly, the results produced in Lemma 3.1 have not been produced in the references mentioned.

Lemma 3.1 Let $\psi$ satisfy condition $\mathcal{M}$. Then $\psi \in W\left(L^{\infty}, l^{1}\right)$, and (3.6) holds. Moreover, the following are equivalent:

$$
\begin{aligned}
& \sum_{j, k \in \mathbb{Z}} \sup _{[0,1)}|\psi|\left(2^{j} x+k\right)<\infty, \\
& \sum_{j, k \in \mathbb{Z}} \sup _{x \in \mathbb{R}}|\psi|\left(2^{j} x+k\right)<\infty, \quad \text { and } \\
& \sum_{j, k \in \mathbb{Z}} \sup _{m, n}|\psi|\left(2^{j} x+k\right)<\infty .
\end{aligned}
$$

The finiteness does not depend on $m$ or $n$. 
Proof of Lemma $3.1 \psi \in W\left(L^{\infty}, l^{1}\right)$ and the part of equivalence are obvious. $\psi \in W\left(L^{\infty}, l^{1}\right)$ leads to $\psi \in L^{p}(\mathbb{R})$, for all $1 \leq p \leq \infty$. Next, we prove (3.6). We set

$$
I_{m, n}^{*}:=\left[2^{-m}(n-1), 2^{-m}(n+2)\right),
$$

and we also note that $\int_{1}^{\infty} \varphi(x) d x<\infty$. For any fixed $(m, n) \in \Omega$,

$$
\begin{aligned}
& \left(\sum_{\substack{j, k \in \mathbb{Z} \\
j \geq m}} \sup _{j I_{m, n}+k}|\psi|(x)\right) \int_{\mathbb{R} \backslash I_{m, n}^{*}} 2^{j / 2}\left|\psi_{j, k}\right| \\
& \quad \leq\left(\sum_{\substack{j, k \in \mathbb{Z} \\
j \geq m}} \sup _{I_{m, n}+k}|\psi|(x)\right)\left(\int_{-\infty}^{2^{-m}(n-1)}+\int_{2^{-m}(n+2)}^{\infty}\right) 2^{j / 2}\left|\psi \psi_{j, k}\right| \\
& \leq\left(\sum_{\substack{j, k \in \mathbb{Z} \\
j \geq m}} \sup _{I_{m, n}+k}|\psi|(x)\right)\left(\int_{-\infty}^{2^{j-m}(n-1)+k}+\int_{2^{j-m}(n+2)+k}^{\infty}\right)|\psi| \\
& \quad=\left(\sum_{\substack{j, k \in \mathbb{Z} \\
j\left[k, k+2^{j-m}\right)}} \sup _{j \geq m}|\psi|(x)\right)\left(\int_{-\infty}^{k-2^{j-m}}+\int_{2^{j-m+1}+k}^{\infty}\right)|\psi|<\infty .
\end{aligned}
$$

The finiteness in (3.8) does not depend on $(m, n)$ and can be proven by breaking $k$ into parts. First,

$$
\begin{aligned}
& \sum_{j=m}^{\infty}\left(\sum_{k \leq-2^{j-m+1}}+\sum_{k>2^{j-m-1}}\right) \sup _{\left[k, k+2^{j-m}\right)}|\psi|(x)\left(\int_{-\infty}^{k-2^{j-m}}+\int_{2^{j-m+1}+k}^{\infty}\right)|\psi| \\
& \quad \leq 2\|\psi\|_{1} \sum_{j=m}^{\infty}\left(\sum_{-\infty}^{k=-2 \cdot 2^{j-m}} \varphi\left(-k-2^{j-m}\right)+\sum_{k=\left[\frac{1}{2} 2^{j-m}\right]+1}^{\infty} \varphi(k)\right) \\
& \quad \leq 4\|\psi\|_{1} \sum_{j=m}^{\infty} \sum_{k=\left[\frac{1}{2} 2^{j-m}\right]+1}^{\infty} \varphi(k) \\
& \quad \leq 4\|\psi\|_{1} \sum_{p=1}^{\infty}\left(\left[\log _{2} p\right]+2\right) \varphi(p) \leq 4\|\psi\|_{1} \int_{1}^{\infty}\left(2+\log _{2} x\right) \varphi(x) d x<\infty
\end{aligned}
$$

Second, applying the Dirichlet test,

$$
\begin{aligned}
& \sum_{j=m}^{\infty}\left(\sum_{-2^{j-m+1}<k \leq 2^{j-m-1}}\right) \sup _{\left[k, k+2^{j-m}\right)}|\psi|(x)\left(\int_{-\infty}^{k-2^{j-m}}+\int_{2^{j-m+1}+k}^{\infty}\right)|\psi| \\
& \quad \leq \sum_{j=m}^{\infty}\left(\sum_{-2^{j-m+1}<k \leq 2^{j-m-1}}\right) \sup _{\left[k, k+2^{j-m}\right)}|\psi|(x)\left(\int_{-\infty}^{-2^{j-m-1}}+\int_{2^{j-m+1}+k}^{\infty}\right)|\psi|<\infty .
\end{aligned}
$$

Because the double summation of (3.10) is finite, (3.9) is finite. We have

$$
\sum_{j>m} \sum_{k} \sup _{\left[k, k+2^{j-m}\right)}|\psi|(x)<\infty
$$


We will prove (3.10) using a pedagogical argument. Assuming that (3.10) does not hold, and given $V>0$, there exist $j_{0}>m, k_{0} \in \mathbb{Z}, q_{1}, q_{2} \in \mathbb{N}$ such that

$$
V<\sum_{j=j_{0}}^{j_{0}+q_{1}-1} \sum_{k=k_{0}}^{k_{0}+q_{2}-1} \sup _{\left.[k, k+2]^{j-m}\right)}|\psi|(x) \leq q_{1} q_{2} \sup _{\left[k_{1}, k_{1}+2^{j_{1}-m}\right)}|\psi|(x),
$$

where $j_{1} \in\left\{j_{0}, j_{0}+1, \ldots, j_{0}+q_{1}-1\right\}, k_{0} \in\left\{k_{0}, k_{0}+1, \ldots, k_{0}+q_{2}-1\right\}$. There also exist $x_{0}$ and $n_{0} \in \mathbb{Z}$ with $x_{0} \in\left[k_{1}, k_{1}+2^{j_{1}-m}\right)$ such that

$$
\begin{aligned}
\frac{V}{q_{1} q_{2}}<|\psi|\left(x_{0}\right) & \leq \sup _{\left[n_{0}, n_{0}+1\right)}|\psi|(x) \\
& \leq \varphi(x) \chi_{\left[n_{1}, n_{1}+1\right)} \leq \varphi(x) \chi_{\left[n_{1}, n_{1}+q_{1} q_{2}\right)},
\end{aligned}
$$

where $n_{1}:=\min \left\{\left|n_{0}\right|,\left|n_{0}+1\right|\right\}$. Performing integration on both sides,

$$
V=\int_{n_{1}}^{n_{1}+q_{1} q_{2}} \frac{V}{q_{1} q_{2}}<\int_{n_{1}}^{n_{1}+q_{1} q_{2}} \varphi(x) .
$$

This is a contradiction to $\int_{0}^{\infty} \varphi(x) d x<\infty$.

Putting $(m, n)=(0,1)$ into (3.7), we obtain

$$
\sum_{\substack{j, k \in \mathbb{Z} \\ j \geq 0}} \sup _{[0,1)}|\psi|(x+k)<\infty
$$

and this leads to

$$
\sum_{\substack{j^{\prime}, k^{\prime} \in \mathbb{Z} 22^{j^{\prime}}[0,1) \\ j^{\prime}<0}} \sup _{\substack{j, k \in \mathbb{Z} \\ j \geq 0}}|\psi|\left(x+k^{\prime}\right) \leq \sup _{\substack{j \\ j}}|\psi|(x+k)<\infty .
$$

So, (3.6) holds.

Next, we prove Theorem 3.2, which explains that the frame operator of the Riesz wavelet basis with the decay condition has $L^{p}$-boundedness. It has to be stressed that Theorem 3.2 does not need to take the behavior of the dual basis into consideration, and it also does not require the dual basis to have a wavelet structure.

Theorem 3.2 The frame operator $S$ of a Riesz wavelet basis $\mathcal{F}_{\psi}$ is of weak type $(1,1)$ and of type $(p, p)$, for all $1<p<\infty$.

Proof of Theorem 3.2 The $L^{p}$-boundedness of $S$ can be proven if $S$ being of weak type $(1,1)$ can be proven. We note that $S$ is of type $(2,2)$. The proof is based on two inequalities:

$$
\begin{aligned}
& \|g\|_{2}^{2} \leq \alpha A_{1}\|f\|_{1} \text { and } \\
& m\{x:|S h|>\alpha / 2\} \leq \frac{B_{1}}{\alpha}\|f\|_{1},
\end{aligned}
$$


where $A_{1}$ and $B_{1}$ do not depend on $f$ or $\alpha$. $S$ being of weak type $(1,1)$ can be proven by the following:

$$
\begin{aligned}
m\{x:|S f|>\alpha\} & \leq m\{x:|S g|>\alpha / 2\}+m\{x:|S h|>\alpha / 2\} \\
& \leq m\left\{x:|S g|^{2}>\alpha^{2} / 4\right\}+\frac{B_{1}}{\alpha}\|f\|_{1} \\
& \leq\left[\left(4 A_{1}+B_{1}\right) / \alpha\right]\|f\|_{1},
\end{aligned}
$$

for all $f \in L^{1} \cap L^{2}(\mathbb{R}), \alpha>0$. Suppose for the moment that we know, and then we will immediately see from Marcinkiewicz interpolation theorem that $S$ is of type $(p, p), 1<p<2$, and thus by duality for all $p, 2<p<\infty$.

First, we claim that the first inequality holds. By Lemma 3.1 and the Dirichlet test, for any $(m, n),\left(m^{\prime}, n^{\prime}\right) \in \Omega$ with $m \leq m^{\prime}$, there exist constants $M_{1}, M_{2}$, and $M_{3}$ which do not depend on $m$ or $n$, such that

$$
\begin{aligned}
& M_{1}:=4\left\|S^{-1}\right\|_{2}^{2}\|\psi\|_{2}^{2}\left[\sum_{\substack{j^{\prime}, k^{\prime} \in \mathbb{Z} \\
j^{\prime}<m^{\prime}}} 2^{\frac{j^{\prime}-m^{\prime}}{2}} \sup _{I_{m^{\prime}, n^{\prime}}}|\psi|\left(2^{j^{\prime}} x+k^{\prime}\right)\right]<\infty, \\
& M_{3}:=\left[\sum_{\substack{j, k \in \mathbb{Z} \\
j<m}} 2^{j-m^{\prime}} \sup _{I_{m^{\prime}, n^{\prime}}}|\psi|\left(2^{j} x+k\right)\right]^{-1}\left[1+\sum_{\substack{j, k \in \mathbb{Z} \\
j<m}} 2^{\frac{j-m^{\prime}}{2}} \sup _{I_{m^{\prime}, n^{\prime}}}|\psi|\left(2^{j} x+k\right)\right]<\infty, \\
& \sum_{\substack{j, k \in \mathbb{Z} \\
j<m}} 2^{\frac{j-m^{\prime}}{2}} \sup _{I_{m, n}}|\psi|\left(2^{j} x+k\right) \leq M_{2} \sum_{\substack{j, k \in \mathbb{Z} \\
j<m}} 2^{\frac{j-m^{\prime}}{2}} \sup _{I_{m^{\prime}, n^{\prime}}}|\psi|\left(2^{j} x+k\right)<\infty, \\
& \sum_{\substack{j, k \in \mathbb{Z} \\
j<m}} 2^{\frac{j-m^{\prime}}{2}} \sup _{I_{m^{\prime}, n^{\prime}}}|\psi|\left(2^{j} x+k\right) \leq M_{3} \sum_{\substack{j, k \in \mathbb{Z} \\
j<m}} 2^{j-m^{\prime}} \sup _{\substack{I_{m^{\prime}, n^{\prime}} \\
j<m}}|\psi|\left(2^{j} x+k\right)<\infty, \\
& \left\|\sum_{(m, n) \in \Omega} P_{m}\left(f \chi_{I_{m, n}}\right)\right\|_{2}^{2} \\
& =\int_{\mathbb{R}} \sum_{(m, n) \in \Omega} \sum_{\left(m^{\prime}, n^{\prime}\right) \in \Omega} P_{m}\left(f \chi_{\left.I_{m, n}\right)} \overline{P_{m^{\prime}}\left(f \chi_{I_{m^{\prime}, n^{\prime}}}\right)}\right. \\
& \leq \sum_{(m, n) \in \Omega} \sum_{\left(m^{\prime}, n^{\prime}\right) \in \Omega} \sum_{\substack{j, k \in \mathbb{Z} \\
j<m}} \sum_{j^{\prime}, k^{\prime} \in \mathbb{Z}}\left|\left\langle f \chi_{j_{m, n}<m^{\prime}}, \psi_{j, k}\right\rangle\left\langle f \chi_{I_{m^{\prime}, n^{\prime}}}, \psi_{j^{\prime}, k^{\prime}}\right\rangle\left\langle S^{-1} \psi_{j, k}, S^{-1} \psi_{j^{\prime}, k^{\prime}}\right\rangle\right| \\
& \leq 2\left\|S^{-1}\right\|_{2}^{2}\|\psi\|_{2}^{2} \sum_{(m, n) \in \Omega} \sum_{\substack{\left(m^{\prime}, n^{\prime}\right) \in \Omega \\
m \leq m^{\prime}, n \leq n^{\prime}, j \in \mathbb{Z}}} \sum_{j<m} \sum_{j j^{\prime}, k^{\prime} \in \mathbb{Z}}\left(\int_{j^{\prime}<m^{\prime}}|f|\right)\left(2^{\frac{j}{2}} \sup _{I_{m, n}}|\psi|\left(2^{j} x+k\right)\right) \\
& \times\left(\left(2 \cdot 2^{-m^{\prime}} \alpha\right) 2^{j^{\prime}} \sup _{I_{m^{\prime}, n^{\prime}}}|\psi|\left(2^{j^{\prime}} x+k^{\prime}\right)\right) \\
& \leq 4 \alpha\left\|S^{-1}\right\|_{2}^{2}\|\psi\|_{2}^{2}\left(\sum_{(m, n) \in \Omega} \int_{I_{m, n}}|f|\right) \sum_{\substack{\left(m^{\prime}, n^{\prime}\right) \in \Omega \\
m \leq m^{\prime}}} \sum_{\substack{j, k \in \mathbb{Z} \\
j<m}}\left(2^{\frac{j-m^{\prime}}{2}} \sup _{I_{m, n}}|\psi|\left(2^{j} x+k\right)\right)
\end{aligned}
$$




$$
\begin{aligned}
& \times \sum_{\substack{j^{\prime}, k^{\prime} \in \mathbb{Z} \\
j^{\prime}<m^{\prime}}}\left(2^{\frac{j^{\prime}-m^{\prime}}{2}} \sup _{I_{m^{\prime}, n^{\prime}}}|\psi|\left(2^{j^{\prime}} x+k^{\prime}\right)\right) \\
& \leq \alpha M_{1} M_{2} M_{3}\left(\sum_{(m, n) \in \Omega} \int_{I_{m, n}}|f|\right) \sum_{\substack{\left(m^{\prime}, n^{\prime}\right) \in \Omega \\
m \leq m^{\prime} \\
m \leq k \in \mathbb{Z} \\
j<m}} 2^{j-m^{\prime}} \sup _{I_{m^{\prime}, n^{\prime}}}|\psi|\left(2^{j} x+k\right) \\
& \leq \alpha M_{1} M_{2} M_{3}\left(\sum_{(m, n) \in \Omega} \int_{I_{m, n}}|f|\right)\left(\sum_{p \in \mathbb{Z}} \sum_{m^{\prime}=m}^{\infty} \sum_{\substack{n^{\prime}=p 2^{m^{\prime}-m} \\
\text { (p+1) } 2^{m^{\prime}-m}+1}} \sum_{\substack{j, k \in \mathbb{Z} \\
j<m}} 2^{j-m^{\prime}} \sup _{I_{m^{\prime}, n^{\prime}}}|\psi|\left(2^{j} x+k\right)\right. \\
& \leq \alpha M_{1} M_{2} M_{3}\left(\sum_{(m, n) \in \Omega} \int_{I_{m, n}}|f|\right) \sum_{\substack { p \in \mathbb{Z} \\
\begin{subarray}{c}{j, k \in \mathbb{Z} \\
j<m{ p \in \mathbb { Z } \\
\begin{subarray} { c } { j , k \in \mathbb { Z } \\
j < m } }\end{subarray}} 2^{j-m} \sup _{I_{m, p}}|\psi|\left(2^{j} x+k\right) \leq \alpha N\|f\|_{1},
\end{aligned}
$$

where, by the Dirichlet test,

$$
N:=M_{1} M_{2} M_{3} \sum_{p \in \mathbb{Z}} \sum_{\substack{j, k \in \mathbb{Z} \\ j<m}} 2^{j-m} \sup _{\substack{m, p \\ j<}}|\psi|\left(2^{j} x+k\right)<\infty
$$

The finiteness of $N$ derives from:

(1) Recall that $\left\{I_{m^{\prime}, n^{\prime}}\right\}_{\left(m^{\prime}, n^{\prime}\right) \in \Omega}$ are disjoint. Under $m \leq m^{\prime}$ and $p 2^{m^{\prime}-m} \leq n^{\prime}<(p+1) 2^{m^{\prime}-m}$, we have

$$
I_{m^{\prime}, n^{\prime}} \subset I_{m, p} .
$$

It leads, for each $p$, to

$$
\sum_{m^{\prime}=m}^{\infty} \sum_{n^{\prime}=p 2^{m^{\prime}-m}}^{(p+1) 2^{m^{\prime}-m}-1} 2^{-m^{\prime}}<2^{-m}
$$

(2) For any finite terms of $p, j$, and $k$,

$$
\sum_{\substack{p, j, k \\ j<m}} \sup _{I_{m, p}}|\psi|\left(2^{j} x+k\right) \leq \sum_{\text {finite } k^{\prime}} \sup _{\substack{ \\j(0,1)}}|\psi|\left(x+k^{\prime}\right)<\|\psi\|_{W\left(L^{\infty}, l^{1}\right)}<\infty .
$$

Next,

$$
\int_{\mathbb{R}}\left|f \chi_{F}\right|^{2} \leq \alpha \int_{F}|f| \leq \alpha\|f\|_{1}
$$

and it leads to $\|g\|_{2}^{2} \leq(N+1) \alpha\|f\|_{1}$.

For the second inequality, we set

$$
\begin{aligned}
& I_{m, n}^{*}:=\left[2^{-m}(n-1), 2^{-m}(n+2)\right), \quad F^{*}:=\mathbb{R} \backslash \bigcup_{(m, n) \in \Omega} I_{m, n}^{*}, \\
& B_{1}^{\prime}:=\|\psi\|_{1} \sum_{\substack{j, k \in \mathbb{Z} \\
j \geq m}} \sup _{m, n}|\psi|\left(2^{j} x+k\right),
\end{aligned}
$$




$$
\begin{aligned}
\int_{F^{*}}|S h| & \leq \int_{\mathbb{R} \backslash I_{m, n}^{*}}|S h| \\
& \leq \sum_{\substack {(m, n) \in \Omega \\
\begin{subarray}{c}{j, k \in \mathbb{Z} \\
j \geq m{ ( m , n ) \in \Omega \\
\begin{subarray} { c } { j , k \in \mathbb { Z } \\
j \geq m } }\end{subarray}}\left|\left\langle f \chi_{I_{m, n}}, \psi_{j, k}\right\rangle\right|\left(\int_{\mathbb{R} \backslash I_{m, n}^{*}}\left|\psi_{j, k}\right|\right) \\
& \leq\|\psi\|_{1}\left(\sum_{(m, n) \in S} \int_{I_{m, n}}|f|\right)\left(\sum_{\substack{j, k \in \mathbb{Z} \\
j \geq m}} \sup _{\substack{I_{m, n} \\
j}}|\psi|\left(2^{j} x+k\right)\right) \\
& \leq B_{1}^{\prime}\|f\|_{1} .
\end{aligned}
$$

Finally, we note that $m\left\{\mathbb{R} \backslash F^{*}\right\}<3 / \alpha\|f\|_{1}$ and thus

$$
m\{x:|S h|>\alpha / 2\} \leq\left[\left(2 B_{1}^{\prime}+3\right) / \alpha\right]\|f\|_{1} .
$$

Theorem 3.3 gives a characterization on a pair of biorthogonal Riesz wavelet bases for Lebesgue spaces. The proof relies on the $L^{p}$-boundedness of the frame operator of the dual wavelet basis which is an application from Theorem 3.2.

Theorem 3.3 Let $\mathcal{F}_{\psi}$ and $\mathcal{F}_{\widetilde{\psi}}$ be a pair of biorthogonal Riesz wavelet bases for $L^{2}(\mathbb{R})$. Then:

(1) The operator $H$ is of weak type $(1,1)$ and of type $(p, p)$, for all $1<p<\infty$. Moreover,

$$
\begin{aligned}
& C^{\prime}\|f\|_{p} \leq\|S f\|_{p} \leq C^{\prime \prime}\|f\|_{p}, \\
& C_{1}^{\prime}\|f\|_{p} \leq\|H f\|_{p} \leq C_{1}^{\prime \prime}\|f\|_{p},
\end{aligned}
$$

for all $f \in L^{p}(\mathbb{R})$ and some constants $C^{\prime}, C^{\prime \prime}, C_{1}^{\prime}, C_{1}^{\prime \prime}$.

(2) The two operators $S$ and $H$ are bijective on $L^{p}(\mathbb{R}), 1<p<\infty$.

(3) $\mathcal{F}_{\psi}$ and $\mathcal{F}_{\widetilde{\psi}}$ are also unconditional bases for $L^{p}(\mathbb{R}), 1<p<\infty$.

Proof of Theorem 3.3 (1) Using the same argument as the proof of Theorem 3.2, the $L^{p}$ boundedness of $H$ can be proven. Next, we will prove that $S$ is bounded below. By duality, for all $f^{\prime} \in L^{2} \cap L^{p}(\mathbb{R})$, we have

$$
\begin{aligned}
\left\|f^{\prime}\right\|_{p} & =\sup _{\substack{\|h\|_{q} \leq 1, h \in L^{2} \cap L^{q}(\mathbb{R})}}\left|\int_{\mathbb{R}} f^{\prime} \bar{h}\right| \\
& \leq \sup _{\substack{\|h\|_{q} \leq 1, h \in L^{2} \cap L^{q}(\mathbb{R})}}\left|\left\langle S f^{\prime}, H h\right\rangle_{L^{2}(\mathbb{R})}\right| \\
& \leq \frac{1}{C}\left\|S f^{\prime}\right\|_{p^{\prime}}
\end{aligned}
$$

for some constant $\frac{1}{C}>0$ and $1 / p+1 / q=1$. Since $L^{2} \cap L^{p}(\mathbb{R})$ is dense in $L^{p}(\mathbb{R}), 1<p<\infty$, for all $f \in L^{p}(\mathbb{R})$, there exists $\left\{f_{k}\right\} \subset L^{2} \cap L^{p}(\mathbb{R})$ such that $f_{k}$ converges to $f$ in $L^{p}$-norm,

$$
\|f\|_{p}=\lim _{k \rightarrow \infty}\left\|f_{k}\right\|_{p} \leq \frac{1}{C} \lim _{k \rightarrow \infty}\left\|S f_{k}\right\|_{p}=\frac{1}{C}\|S f\|_{p} .
$$


So, $S$ is injective and has a closed range. Similarly, we can prove that $H$ is bounded below by using the method described above.

(2) To claim $S$ is bijective, that the adjoint of $S$ is bounded below needs to be proven. We denote by $S^{*}$ the adjoint of $S$ which is on $L^{q}(\mathbb{R})$ into $L^{q}(\mathbb{R})$, and which is based on (3.13),

$$
\begin{aligned}
\|f\|_{p} \cdot 1 & \leq \frac{1}{C}\|S f\|_{p}=\frac{1}{C} \sup _{\|g\|_{q}=1}\left|\int_{\mathbb{R}}(S f) g\right| \\
& =\frac{1}{C} \sup _{\|g\|_{q}=1}\left|\int_{\mathbb{R}} f\left(S^{*} g\right)\right| \leq \frac{1}{C}\|f\|_{p}\left\|S^{*} g\right\|_{q} .
\end{aligned}
$$

This leads to

$$
\|g\|_{q}=1 \leq \frac{1}{C}\left\|S^{*} g\right\|_{q}
$$

We can conclude that $S$ is bijective on $L^{p}(\mathbb{R}), 1<p<\infty$. Similarly, we can prove that $H$ is bijective on $L^{p}(\mathbb{R}), 1<p<\infty$ by using the method described above.

(3) In order to prove (3), we will change hypotheses (3.1)-(3.5) to:

$P_{m}$ is the projection onto a subspace of $\mathcal{V}_{m}:=\overline{\operatorname{span}}\left\{\psi_{j, k}: j, k \in \mathbb{Z}, j<m\right\}$,

$$
P_{m}(f):=\sum_{\substack{j, k \in \mathbb{Z} \\ j<m}}\left\langle f, \widetilde{\psi}_{j, k}\right\rangle \psi_{j, k},
$$

for all $f \in L^{1} \cap L^{2}(\mathbb{R})$. We set

$$
g:=f \chi_{F}+\sum_{(m, n) \in \Omega} P_{m}\left(f \chi_{I_{m, n}}\right)
$$

producing

$$
\begin{aligned}
h & :=f-g=\sum_{(m, n) \in \Omega}\left[f \chi_{I_{m, n}}-P_{m}\left(f \chi_{I_{m, n}}\right)\right] \\
& =\sum_{(m, n) \in \Omega}\left[\sum_{j, k \in \mathbb{Z}}\left\langle f \chi_{I_{m, n}}, \widetilde{\psi}_{j, k}\right\rangle \psi_{j, k}-P_{m}\left(f \chi_{I_{m, n}}\right)\right] \\
& =\sum_{\substack {(m, n) \in \Omega \\
\begin{subarray}{c}{j, k \in \mathbb{Z} \\
j \geq m{ ( m , n ) \in \Omega \\
\begin{subarray} { c } { j , k \in \mathbb { Z } \\
j \geq m } }\end{subarray}}\left\langle f \chi_{I_{m, n}}, \widetilde{\psi}_{j, k}\right\rangle \psi_{j, k} .
\end{aligned}
$$

Define an operator $Q$ on $L^{2}(\mathbb{R})$ as

$$
Q(\cdot):=\sum_{j, k \in \mathbb{Z}} \epsilon_{j, k}\left\langle\cdot, \widetilde{\psi}_{j, k}\right\rangle \psi_{j, k}
$$

for all $\epsilon_{j, k}= \pm 1$. Using a similar argument to Theorem 3.2, the $L^{p}$-boundedness of $Q$ can be proven. Secondly, since $L^{2} \cap L^{p}(\mathbb{R})$ is a dense subset of $L^{p}(\mathbb{R}), 1<p<\infty$, we have $f=\sum_{j, k \in \mathbb{Z}}\left\langle f, \widetilde{\psi}_{j, k}\right\rangle \psi_{j, k} \in L^{p}(\mathbb{R})$, for any $f \in L^{2} \cap L^{p}(\mathbb{R})$. Thus, for each $f \in L^{p}(\mathbb{R})$, the series $\sum_{j, k \in \mathbb{Z}}\left\langle f, \widetilde{\psi}_{j, k}\right\rangle \psi_{j, k}$ converges unconditionally (since $Q$ is of type $\left.(p, p)\right)$ in $L^{p}(\mathbb{R})$ so that 
it must converge to $f$. Similarly, we can prove that $\mathcal{F}_{\widetilde{\psi}}$ is an unconditional basis of $L^{p}(\mathbb{R})$, $1<p<\infty$ by using the methods described above.

In order to fully understand the main result, we need some basic terminology. A prewavelet basis (semi-orthogoal basis) is orthogonal between the different dilation levels and all dilations and shifts form a Riesz basis for $L^{2}(\mathbb{R})$. This was first used by Battle [13]. More information can be found in [14]. Wiener's lemma was used to prove Theorem 3.4. Let $\mathcal{A}$ be the set of all formal power series $f=\sum_{k \in \mathbb{Z}} a_{k} z^{k}$ for which

$$
\|f\|_{\mathcal{A}}:=\sum_{k \in \mathbb{Z}} \sigma(k)\left|a_{k}\right|<\infty
$$

where $\sigma$ is the weight function which satisfies $\sigma(x+y) \leq \sigma(x) \sigma(y)$. For example, $\sigma(x)=$ $(1+x)^{p}, p>1$. So, if $\left\{\sigma(k) a_{k}\right\} \in l^{1}(\mathbb{Z}), f$ is a continuous function of $z$ on the torus $\mathbb{T}$, and $\mathcal{A}$ is a commutative Banach algebra with the unit. In our application, we denote

$$
[f, g](z):=\sum_{k \in \mathbb{Z}}(f * g)(k) z^{k}
$$

where the sequence $f * g$ on $\mathbb{Z}$ is defined by

$$
(f * g)(k):=\int_{\mathbb{R}} f(x) \bar{g}(x-k) d x=\int_{\mathbb{R}} f(x+k) \bar{g}(x) d x .
$$

Now, we are ready to prove Theorem 3.4.

Theorem 3.4 Let $\psi$ be a pre-wavelet of $L^{2}(\mathbb{R})$ with

$$
\|\sigma \psi\|_{w}:=\sum_{a \in \mathbb{Z}} \sup _{[0,1)}|\sigma \psi|(x+a)<\infty
$$

Then, we have the following.

(1) The dual basis $\left\{\widetilde{\psi}_{j, k}: j, k \in \mathbb{Z}\right\}$ of $\left\{\psi_{j, k}: j, k \in \mathbb{Z}\right\}$ has a wavelet structure, and the generator $\tilde{\psi}$ satisfies

$$
\|\sigma \widetilde{\psi}\|_{w} \leq\|\sigma \psi\|_{w}\|\widetilde{\psi}\|_{\mathcal{A}}<\infty
$$

(2) The two operators $S$ and $H$ are bijective on $L^{p}(\mathbb{R}), 1<p<\infty$. Both $\left\{\psi_{j, k}: j, k \in \mathbb{Z}\right\}$ and $\left\{\widetilde{\psi}_{j, k}: j, k \in \mathbb{Z}\right\}$ are also unconditional bases for $L^{p}(\mathbb{R}), 1<p<\infty$.

Proof of Theorem 3.4 (1) This proof is presented in two parts. First, we want to describe the form of the dual basis. Second, we will prove $\left\{\widetilde{\psi}_{j, k}: j, k \in \mathbb{Z}\right\}$ is, indeed, the dual basis. We have

$$
\begin{aligned}
\sum_{k \in \mathbb{Z}} \sigma(k)|(\psi * \bar{\psi})|(k) & \leq \sum_{l \in \mathbb{Z}} \sum_{k \in \mathbb{Z}} \sigma(k) \int_{[0,1)}|\psi|(x+l+k)|\psi|(x+l) d x \\
& \leq \sum_{l \in \mathbb{Z}}\left[\sup _{[0,1)}|\sigma \psi|(x+l) \sum_{k \in \mathbb{Z}} \sup _{[0,1)}|\sigma \psi|(x+l+k)\right] \\
& \leq\|\sigma \psi\|_{w}^{2}<\infty .
\end{aligned}
$$


This leads to $[\psi, \psi] \in \mathcal{A}$. Next, since

$$
\int_{0}^{1}\left(\sum_{k \in \mathbb{Z}}|\psi|(x+k)\right)^{2} d x \leq\|\psi\|_{w}^{2},
$$

we have

$$
[\psi, \psi]\left(e^{-i \xi}\right)=\sum_{k \in \mathbb{Z}}|\widehat{\psi}(\xi+2 \pi k)|^{2}>0
$$

and thus $[\psi, \psi](z)>0$, for all $z \in \mathbb{T}[14]$, Theorem 3.2. Let $a$ be a sequence given by

$$
a_{k}:=\psi * \bar{\psi}(k)
$$

which leads to $\sum_{k \in \mathbb{Z}} a_{k} z^{k} \in \mathcal{A}$. Using Wiener's lemma, there exists $\left\{c_{k}\right\}_{k \in \mathbb{Z}} \in l^{1}(\mathbb{Z})$ for which the formal power series

$$
\frac{1}{[\psi, \psi]}(z)=\sum_{k \in \mathbb{Z}} c_{k} z^{k} \in \mathcal{A}
$$

We denote $\widetilde{\psi}:=\sum_{k \in \mathbb{Z}} c_{k} \psi(x+k)$ and consider the Cauchy product

$$
\begin{aligned}
\sum_{a \in \mathbb{Z}} \sup _{[0,1)}|\sigma \widetilde{\psi}|(x+a) & \leq \sum_{a, k \in \mathbb{Z}}\left|\sigma(k) c_{k}\right| \sup _{[0,1)}|\sigma \psi|(x+a+k) \\
& \leq\|\sigma \psi\|_{w}\|\widetilde{\psi}\|_{\mathcal{A}}<\infty .
\end{aligned}
$$

Thus, we have $\|\sigma \widetilde{\psi}\|_{w}<\infty$, and

$$
[\widetilde{\psi}, \psi](z)=\frac{1}{[\psi, \psi]}(z) \cdot[\psi, \psi](z)=1,
$$

for all $z \in \mathbb{T}$. Finally, we will explain that $\left\{\widetilde{\psi}_{j, k}\right\}$ is the dual basis of $\left\{\psi_{j, k}\right\}$. For this there are three reasons. The first one is that a pre-wavelet basis is orthogonal between the different dilation levels and all dilations and shifts form a Riesz basis for $L^{2}(\mathbb{R})$. Second, from (3.14) and (3.15), (3.17) is equivalent to

$$
\langle\widetilde{\psi}, \psi(\cdot-\alpha)\rangle=\delta_{0 \alpha}, \quad \text { for all } \alpha \in \mathbb{Z} .
$$

Combining two reasons, we can conclude that $\left\{\widetilde{\psi}_{j, k}\right\}$ and $\left\{\psi_{j, k}\right\}$ are biorthogonal. The last one is that $\left\{\psi_{j, k}\right\}$ has a unique dual basis.

(2) We have constructed the dual basis of $\left\{\psi_{j, k}\right\}$ in (1), and $\left\{\widetilde{\psi}_{j, k}\right\}$ has the same decay as $\left\{\psi_{j, k}\right\}$. Applying that and Theorem 3.3, we complete the proof of (2).

\section{Further remarks and examples}

In this section, we give comments and examples. Bownik and Weber point out the connection between the behavior of a canonical dual of $\left\{\psi_{j, k}\right\}$ and how $\psi$ generates a GMRA [15]. For a Riesz wavelet $\psi$, the shift invariance of negative dilates $V_{0}(\psi)$ implies a wavelet structure of the canonical dual. They have also given an interesting example of a wavelet 
frame for which the canonical dual does not have the wavelet structure, but other dual frames with the wavelet structure exist. Bownik and Lemvig also mentioned in another paper [8] that a frame may have an infinite number of dual frames with a wavelet structure. The last paper [16] we refer to was written by Lemvig; he constructed pairs of dual band-limited wavelet frames.

The aforementioned three papers all depend on one important theorem [6], p.277, Theorem 12.1.3 (or [17], p.263, [16], Theorem 2.1). Part of the results are generated by using the multiresolution analysis (MRA). However, Theorem 3.4 provides a method to construct the dual wavelet of a given pre-wavelet without using the mentioned theorem or MRA. Through the fact that dual basis has wavelet structure and it has the same decay as basis, less limitations are applied on wavelets to accomplish our results.

Next, we review some typical pre-wavelets and biorthogonal wavelets.

(I) Compactly supported. Compactly supported wavelets certainly satisfy condition $\mathcal{M}$ and thus Theorem 3.3 can be applied to all compactly supported orthogonal wavelets. The earliest example is the Haar wavelet [18], Chapter 1, Example A. For non-orthogonal cases, Chui and Wang [19], and independently Jia and Micchelli [14] have constructed compactly supported pre-wavelets. Riemenschneider considered a cardinal spline approach to construct compactly supported, skew-symmetric pre-wavelets [20,21]. Cohen et al. have constructed biorthogonal wavelets that are symmetric, regular, and compactly supported [7].

(II) Decay. Theorems 3.3-3.4 can be applied to many mild decay wavelets. For example, Daubechies' wavelets [22], Lemarié-Meyer wavelets [23, 24], and spline wavelets of high order (orthogonal [25, 26], semi-orthogoanl [19, 27], biorthogonal [7]). In addition, we would like to emphasize an important fact. Lemma 3.1 guarantees that $\varphi$ is a radial decreasing $L^{1}$-majorant of $\psi$ [18], p.218, Chapter 5. Indeed, the boundedness of $\varphi$ follows from

$\varphi$ is decreasing on $[0, \infty)$, and $|\psi|(x) \leq \varphi(|x|)$

for almost every $x \in \mathbb{R}$. $\varphi$ contrasts with typical majorants of wavelets (polynomial $C(1+$ $x)^{-\alpha}, \alpha>1$, exponential $D e^{-\beta x}, \beta>0$ ) by stressing that the majorant $\varphi$ is generated by wavelets themselves, not borrowed from specific $L^{1}$ functions. Wojtaszczyk reported that orthogonal wavelet bases are unconditional bases for Lebesgue spaces by providing some assumptions [12]. His assumptions seem verbose in comparison to condition $\mathcal{M}$.

Unser has constructed pre-wavelets in [28] that these wavelets converge to a cosinemodulated Gaussian function as the degree of the spline goes to infinity. Another remarkable wavelet was found in [29] which has subexponential decay $\left(\psi(x) \leq E_{\gamma} e^{-|x|^{1-\gamma}}\right.$, $0<\gamma<1, x \in \mathbb{R}$ ), it is band-limited and belongs to $C^{\infty}$.

For different methods to construct dual wavelets, Kim has shown that if a Riesz wavelet is associated with an MRA, then it has a dual Riesz wavelet [30], Corollary 2.9. Later, he gave a characterization on biorthogonal/semi-orthogonal wavelets associated with MRA [31]. Combining these two facts, lots of biorthogonal/semi-orthogonal wavelets can be found.

(III) Smooth. Daubechies' wavelets and biorthogonal wavelets in [7] are typical smooth wavelets. References [32], p.856, Theorem 3.3, [33, 34], [35], p.295, Theorems 9.1.5-9.1.6, [18], Chapter 5, Theorems 6.14, 6.23, [2], Chapter 6, and [36], Section 7.3, Theorem 1, ensure that orthogonal wavelets that are sufficiently smooth are unconditional bases for 
Lebesgue spaces and the associated frame operators have $L^{p}$-boundedness. Bui and Laugesen have proven that the frame operator of a wavelet frame (not necessarily a wavelet basis) is bijective on Lebesgue spaces if some assumptions are provided [37]. Mainly $\psi$ and $\psi^{\prime}$ need to have sufficient decay on the frequency domain. The aforementioned results were obtained by using the technique of Calderón-Zygmund operators. Apparently, the method suffers from the requirement of smoothness on wavelets (and thus they do not support the Haar wavelet). In contrast, smoothness is not required for Theorems 3.2-3.4 to hold.

\section{Competing interests}

The authors declare that there is no conflict of interests regarding the publication of this article.

\section{Authors' contributions}

All authors contributed equally to the manuscript and read and approved the final manuscript.

\section{Author details}

'Department of Mechanical Engineering, Nan-Kai University of Technology, Zhongzheng Road, Nantou, 54243, Taiwan.

${ }^{2}$ Ph.D. Program in Mechanical and Aeronautical Engineering, Feng-Chia University, Wenhwa Road, Taichung, 40724,

Taiwan. ${ }^{3}$ Department of Applied Mathematics, Feng-Chia University, Wenhwa Road, Taichung, 40724, Taiwan.

\section{Acknowledgements}

The many suggestions and detailed corrections of anonymous referees are gratefully acknowledged. The authors thank language editor Weilling Chen who has made a significant revision of the manuscript.

Received: 24 April 2015 Accepted: 1 September 2015 Published online: 17 September 2015

\section{References}

1. Tao, $\mathrm{T}: L^{p}$ non-invertibility of frame operators. http://www2.math.ucla.edu/ tao/preprints/Expository/inverse.tex

2. Meyer, Y: Wavelets and Operators. Cambridge University Press, Cambridge (1992)

3. Singer, I: Bases in Banach Spaces I and II. Springer, New York (1970)

4. Wiener, N: Tauberian theorems. Ann. Math. (2) 33, 1-100 (1932)

5. Stein, EM: Singular Integrals and Differentiability Properties of Functions. Princeton University Press, Princeton (1970)

6. Christensen, O: An Introduction to Frames and Riesz Bases. Birkhäuser, Boston (2003)

7. Cohen, A, Daubechies, I, Feauveau, JC: Biorthogonal bases of compactly supported wavelets. Commun. Pure Appl. Math. 45(5), 485-560 (1992)

8. Bownik, M, Lemvig, J: The canonical and alternate duals of a wavelet frame. Appl. Comput. Harmon. Anal. 23(2), 263-272 (2007)

9. Chui, CK, Shi, XL: Inequalities of Littlewood-Paley type for frames and wavelets. SIAM J. Math. Anal. 24(1), 263-277 (1993)

10. Daubechies, I, Han, B: The canonical dual frame of a wavelet frame. Appl. Comput. Harmon. Anal. 12(3), 269-285 (2002)

11. Gripenberg, G: Wavelet bases in $L^{p}(\mathbb{R})$. Stud. Math. 106(2), 175-187 (1993)

12. Wojtaszczyk, P: Wavelets as unconditional bases in $L^{P}(\mathbb{R})$. J. Fourier Anal. Appl. 5(1), 73-85 (1999)

13. Battle, G: A Cardinal spline interpolation and the block spin construction of wavelets. In: Chui, CK (ed.) Wavelets: A Tutorial in Theory and Applications. Academic Press, New York (1992)

14. Jia, RQ, Micchelli, CA: Using the refinement equations for the construction of pre-wavelets II: powers and two. In: Curves and surfaces. Pap. Int. Conf., Chamonix-Mont-Blanc/Fr., 1990, pp. 209-246 (1991)

15. Bownik, M, Weber, E: Affine frames, GMRA's, and the canonical dual. Stud. Math. 159(3), 453-479 (2003)

16. Lemvig, J: Constructing pairs of dual bandlimited framelets with desired time localization. Adv. Comput. Math. 30(3), 231-247 (2009)

17. Chui, CK, Shi, XL: Orthonormal wavelets and tight frames with arbitrary real dilations. Appl. Comput. Harmon. Anal. 9(3), 243-264 (2000)

18. Hernández, E, Weiss, G: A First Course on Wavelets. CRC Press, Boca Raton (1996)

19. Chui, CK, Wang, JZ: On compactly supported spline wavelets and a duality principle. Trans. Am. Math. Soc. 330(2), 903-915 (1992)

20. Riemenschneider, S, Shen, ZW: Box splines, cardinal series, and wavelets. In: Chui, CK (ed.) Approximation Theory and Functional Analysis, pp. 133-149. Academic Press, Boston (1991)

21. Riemenschneider, SD, Shen, Z: Wavelets and pre-wavelets in low dimensions. J. Approx. Theory 71(1), 18-38 (1992)

22. Daubechies, I: Orthogonal bases of compactly supported wavelets. Commun. Pure Appl. Math. 41, 909-996 (1988)

23. Battle, G: A block spin construction of ondelettes. Part I: Lemarié functions. Commun. Math. Phys. 110, 601-615 (1987)

24. Lemarié, PG: Ondelettes à localisation exponentielles. J. Math. Pures Appl. 67(3), 227-236 (1988)

25. Mallat, SG: Multiresolution approximations and wavelet orthogonal bases of $L^{2}(\mathbb{R})$. Trans. Am. Math. Soc. 315(1), 69-87 (1989)

26. Mallat, SG: A theory of multiresolution signal decomposition: the wavelet representation. IEEE Trans. Pattern Anal. Mach. Intell. 11(7), 674-693 (1989)

27. Unser, M, Aldroubi, A, Eden, M: A family of polynomial spline wavelet transforms. Signal Process. 30(2), 141-162 (1993)

28. Unser, M, Aldroubi, A, Eden, M: On the asymptotic convergence of B-spline wavelets to Gabor functions. IEEE Trans. Inf. Theory 38(2), 864-872 (1992) 
29. Dziubański, J, Hernández, E: Band-limited wavelets with subexponential decay. Can. Math. Bull. 41(4), 398-403 (1998)

30. Kim, HO, Kim, RY, Lim, JK: Characterizations of biorthogonal wavelets which are associated with biorthogonal multiresolution analyses. Appl. Comput. Harmon. Anal. 11, 263-272 (2001)

31. Kim, HO, Kim, RY, Lim, JK: Semi-orthogonal frame wavelets and frame multi-resolution analyses. Bull. Aust. Math. Soc. 65(1), 35-44 (2002)

32. Ahmad, $K$, Kumar, R, Debnath, L: Existence of unconditional wavelet packet bases for the spaces $L^{p}(\mathbb{R})$ and $H^{1}(\mathbb{R})$. Taiwan. J. Math. 10(4), 851-863 (2006)

33. Chui, CK, Shi, XL: On $L^{P}$-boundedness of affine frame operators. Indag. Math. 4(4), 431-438 (1993)

34. Chui, CK, Shi, XL: Affine operators and frames of multivariate wavelets. In: Dikshit, HP, Micchelli, CA (eds.) Advances in Computational Mathematics, pp. 139-156. World Scientific, Singapore (1994)

35. Daubechies, I: Ten Lectures on Wavelets. SIAM, Philadelphia (1992)

36. Meyer, Y, Coifman, R: Wavelets: Calderón-Zygmund and Multilinear Operators. Cambridge University Press, Cambridge (1997)

37. Bui, HQ, Laugesen, RS: Wavelet frame bijectivity on Lebesgue and Hardy spaces. J. Fourier Anal. Appl. 19(2), 376-409 (2013)

Submit your manuscript to a SpringerOpen ${ }^{\circ}$ journal and benefit from:

- Convenient online submission

- Rigorous peer review

- Immediate publication on acceptance

- Open access: articles freely available online

- High visibility within the field

- Retaining the copyright to your article 